\title{
MODEL THEORIES WITH TRUTH VALUES IN A UNIFORM SPACE
}

BY C. C. CHANG AND H. JEROME KEISLER

Communicated by J. W. Green, November 25, 1961

In recent years the ultraproduct construction has been applied, e.g. in [4] and [2], to obtain a series of results in the theory of models for the ordinary two-valued first-order predicate logic. Most of the results in [4] and [2] have been generalized in [1] to predicate logic with truth values in the closed real unit interval. In this note we shall see that many of the methods and results of [4] and [2] and [1] can actually be extended to a very wide class of many-valued predicate logics, with truth values in any reasonably well-behaved compact Hausdorff uniform space.

We shall give a detailed statement of the definitions and two representative theorems. A complete account of the theory, including a number of generalizations of theorems from [2] and [1], as well as proofs, will appear in a future publication.

Let $L$ be a formal system with the following symbols: a denumerable set $V$ of individual variables, a set $P$ of finitary predicates, a set $C$ of finitary sentential connectives, a set $Q$ of quantifier symbols, and distinguished symbols $e \in P, \& \in C, \exists \in Q$, where $e$ and $\&$ are binary. Let the set $F$ of formulas be the least set $H$ such that

(i) $\left\{p\left(v_{1}, \cdots, v_{n}\right) \mid p \in P, p\right.$ is $n$-ary, $\left.v_{1}, \cdots, v_{n} \in V\right\} \subseteq H$;

(ii) $\left\{c\left(\phi_{1}, \cdots, \phi_{k}\right) \mid c \in C, c\right.$ is $k$-ary, $\left.\phi_{1}, \cdots, \phi_{k} \in H\right\} \subseteq H$;

(iii) $\{q v(\phi) \mid q \in Q, v \in V, \phi \in H\} \subseteq H$.

Free variables are defined as usual. $\phi$ is a sentence if $\phi \in F$ and $\phi$ has no free variables.

Given sets $X, Y$, and $Z, S(X)$ shall denote the set of all subsets of $X$ and $f: Y \rightarrow Z$ shall mean $f$ is a function on $Y$ into $Z$.

If $X$ is a uniform space with uniformity $u$ (see [3]), a set function $g: S(X) \rightarrow X$ is uniformly continuous if for each $U \in \mathcal{U}$, there exists $U^{\prime} \in \mathcal{U}$ such that whenever $Y \subseteq X \cap U^{\prime}[Z]$ and $Z \subseteq X \cap U^{\prime}[Y]$, then $(g(Y), g(Z)) \in U . \mathfrak{X}=(X, f, t, \hat{c}, \hat{q})_{c \in C, q \in Q}$ is a model theory if

(i) $X$ is a compact Hausdorff uniform space;

(ii) $f, t \in X$ and $f \neq t$;

(iii) for each $k$-ary $c \in C, \hat{c}: X^{k} \rightarrow X$ and $\hat{c}$ is continuous;

(iv) for each $q \in Q, \hat{q}: S(X) \rightarrow X$ and $\hat{q}$ is uniformly continuous. $\mathfrak{U}=\left(A, p_{\mathfrak{A}}\right)_{p \in P}$ is a structure over $X$ if

(i) $A \neq 0$;

(ii) for each $n$-ary $p \in P$, pr: $A^{n} \rightarrow X$;

(iii) for $a, b \in A, e_{\mathfrak{X}}(a, b)=t$ if $a=b$, and $e_{\mathfrak{A}}(a, b)=f$ if $a \neq b$. 
Two structures $\mathfrak{A}$ and $\mathfrak{B}$ are isomorphic, in symbols $\mathfrak{A} \cong \mathfrak{B}$, if there is a 1-1 function $h$ on $A$ onto $B$ such that for every $n$-ary $p \in P$ and all elements $a_{1}, \cdots, a_{n} \in A, p_{\mathfrak{N}}\left(a_{1}, \cdots, a_{n}\right)=p_{\mathfrak{B}}\left(h a_{1}, \cdots, h a_{n}\right)$.

For each $\phi \in F$ and $a: V \rightarrow A$, the value $\operatorname{Val}(\phi, \mathfrak{A}, a) \in X$ is defined inductively in the following manner:

(i) for each $n$-ary $p \in P$,

$$
\operatorname{Val}\left(p\left(v_{1}, \cdots v_{n}\right), \mathfrak{R}, a\right)=p \mathfrak{q}\left(a\left(v_{1}\right), \cdots, a\left(v_{n}\right)\right) ;
$$

(ii) for each $k$-ary $c \in C$, and each $\phi_{1}, \cdots, \phi_{k} \in F$,

$$
\operatorname{Val}\left(c\left(\phi_{1}, \cdots, \phi_{k}\right), \mathfrak{A}, a\right)=\hat{\imath}\left(\operatorname{Val}\left(\phi_{1}, \mathfrak{A}, a\right), \cdots, \operatorname{Val}\left(\phi_{k}, \mathfrak{A}, a\right)\right) \text {; }
$$

(iii) for each $q \in Q, \phi \in F$, and $v \in V, \operatorname{Val}(q v(\phi), \mathfrak{A}, a)=\hat{q}(Y)$ where

$$
Y=\{\operatorname{Val}(\phi, \mathfrak{A}, b) \mid b: V \rightarrow A \text { and } b(u)=a(u) \text { whenever } u \neq v\} \text {. }
$$

Two structures $\mathfrak{A}$ and $\mathfrak{B}$ are equivalent, in symbols $\mathfrak{A} \equiv \mathfrak{B}$, if for every sentence $\phi, \operatorname{Val}(\phi, \mathfrak{A})=\operatorname{Val}(\phi, \mathfrak{B})$.

Given structures $\mathfrak{A}_{i}=\left(A_{i}, p_{i}\right)_{p \in P}$, with $i \in I$, and an ultrafilter $D$ on $I$, the set $A=\prod_{i \in I} A_{i} / D$ is defined as usual. Namely, for each function $f \in \prod_{i \in I} A_{i}$, we write

$$
f / D=\left\{g \in \prod_{i \in I} A_{i} \mid\{i \in I \mid f(i)=g(i)\} \in D\right\},
$$

and we define

$$
\prod_{i \in I} A_{i} / D=\left\{f / D \mid f \in \prod_{i \in I} A_{i}\right\} .
$$

The ultraproduct $\mathfrak{A}=\prod_{i \in I} \mathfrak{A}_{i} / D$ of the structures $\mathfrak{A}_{i}$, with $i \in I$, is defined as follows: for each $n$-ary $p \in P$ and elements $h_{1} / D, \cdots$, $h_{n} / D, p_{\mathfrak{r}}\left(h_{1} / D, \cdots, h_{n} / D\right)$ is the unique $x \in X$ such that for each neighborhood $N$ of $x,\left\{i \mid p_{i}\left(h_{1}(i), \cdots, h_{n}(i)\right) \in N\right\} \in D$. We let $\mathfrak{A}^{I} / D$ denote the ultrapower of $A$. The following generalizes a theorem in $[4]$ and [1].

Compactness Theorem. Let $\mathfrak{X}$ be a model theory. For each $i \in I$, let $x_{i} \in X$ and $\phi_{i}$ be a sentence. Let $J=\{j \mid j \subseteq I$ and $j$ is finite $\}$. Suppose that whenever $i \in j \in J, \operatorname{Val}\left(\phi_{i}, \mathfrak{A}_{j}\right)=x_{i}$. Then there exists an ultrafilter $D$ on $J$ such that, for each $i \in I, \operatorname{Val}\left(\phi_{i}, \prod_{j \in J} \mathfrak{A}_{j} / D\right)=x_{i}$.

A model theory $\mathfrak{X}$ is good if

(i) $\hat{\&}(x, y)=t$ if and only if $x=y=t$;

(ii) $t \in \hat{\exists}(Y)$ if and only if $t$ is in the closure of $Y$;

(iii) if $x \neq y$ and $\phi \in F$, then there exists $\psi \in F$ such that

$$
\{(\mathfrak{A}, a) \mid \operatorname{Val}(\phi, \mathfrak{A}, a)=x\} \subseteq\{(\mathfrak{A}, a) \mid \operatorname{Val}(\psi, \mathfrak{A}, a)=t\}
$$


and

$$
\{(\mathfrak{A}, a) \mid \operatorname{Val}(\phi, \mathfrak{A}, a)=y\} \cap\{(\mathfrak{A}, a) \mid \operatorname{Val}(\psi, \mathfrak{A}, a)=t\}=0 .
$$

Examples of good model theories: (1) The two element Boolean algebra with the sup operator, $(\{0,1\},+, \cdot,-, 0,1$, Sup), with the discrete topology. (2) The $M V$-algebra on the closed real unit interval with the sup operator, $([0,1],+, \cdot,-, 0,1$, Sup), with the usual topology.

We now generalize a theorem in $[2 ; 1]$.

Assume the generalized continuum hypothesis.

Fundamental Theorem. Let $\mathfrak{X}$ be a good model theory, and $\mathfrak{A}, \mathfrak{B}$ be structures over $\mathfrak{X}$. Then $\mathfrak{A} \equiv \mathfrak{B}$ if and only if $\mathfrak{U}^{I} / D \cong \mathfrak{B}^{I} / D$ for some set $I$ and ultrafilter $D$ on $I$.

\section{REFERENCES}

1. C. C. Chang, Theory of models of infinite valued logic. I, II, III, Notices Amer. Math. Soc. 8 (1961), 68-69.

2. H. J. Keisler, Ultraproducts and elementary classes, Doctoral dissertation, Univ. of California, Berkeley, Calif., 1961. Also to appear in Indag. Math.

3. J. L. Kelley, General topology, Van Nostrand, Princeton, N. J., 1955.

4. Anne C. Morel, D. Scott and A. Tarski, Reduced products and the compactness theorem, Notices Amer. Math. Soc. 5 (1958), 674-675.

University of California, Los Angeles, and Institute for Defense Analyses, Princeton 